\title{
AN IMPLEMENTATION OF SOFTWARE EFFORT DURATION AND COST ESTIMATION WITH STATISTICAL AND MACHINE LEARNING APPROACHES
}

\author{
B. M. G. Prasad \\ Research Scholar, Department of Computer Science and Engineering, \\ Rayalaseema University, Andhra Pradesh, India \\ P. V.S. Sreenivas \\ Professor, Department of Computer Science and Engineering, \\ Sreenidhi Institute of Science and Technology, Telangana, India
}

\begin{abstract}
In software industry estimation of effort, cost (EDC) and duration is a troublesome procedure. The exertion itself is in charge of experiencing trouble in evaluating EDC. In any software estimation process, the preeminent advance is to characterize and comprehends the framework to be assessed. Software cost estimation algorithmic methods are estimating by analogy, expert judgment method, price to win method, topdown method, bottom-up method are developed by researchers in the field of software engineering. Any methods are not superior to the other methods. In fact, the qualities and shortcomings of those strategies are differentiating to one another. Presently there are two types of software EDC models to estimate the software those are statistical approaches and machine learning approaches. Many of the software cost estimation methods follows statistical approaches, which are not having the capability to present causes and strong or accurate results. Machine learning techniques are suitable in software engineering because it produces accurate results, which estimates by training rules and iterations. Machine learning techniques resolve the challenges like developing programs in a computer and to give the effective outputs by using the experience
\end{abstract}

Keywords: SPSS, Project code meter, EDC, Machine learning. 
An Implementation of Software Effort Duration and Cost Estimation with Statistical and Machine Learning Approaches

Cite this Article: B. M. G. Prasad and P. V. S. Sreenivas, An Implementation of Software Effort Duration and Cost Estimation with Statistical and Machine Learning Approaches, International Journal of Computer Engineering and Technology, 10(1), 2019, pp. 81-93.

http://iaeme.com/Home/issue/IJCET?Volume=10\&Issue=1

\section{INTRODUCTION}

Software Engineering is a discipline which is majorly concerned about the development of well-organized large software applications that are used in digital computers. This includes various theories and methodologies in planning which includes not only scientific issues like tools and technologies for trained software development but also administration aspects like scheduling, staffing, organizing, planning, controlling and budgeting. Software Engineering is a difficult process which solves the problem by creating it as an abstract model or also related to as an iconic model. When compared with science SE is closely associated with good clients in business. This well-perceived scientific method [8] of software development takes user specification as input of a software produce is released as an output.

This methodical and prearranged advance in software expansion was completed with a sequence of phases referred to as Software Development Life Cycle [5]. Most of popular and generally used SDLC models are agile construction, waterfall model, V model, feature-driven development model, several hybrid models, object-oriented models, and various prototyping models. The structured set of tasks that are incorporated to expand software scheme is called as a procedure. Every phase in SDLC is a development requiring resources, constraints and other tasks. They also impose agreement and help the programmers to improve the exercises that are related to this.

The degrees incorporate social event important from a customer, a point by point practical spec about what the strategy does and whether all the client prerequisites are satisfied. At the point when the customer acknowledges the useful detail according to the example determination the product is being created and tried. The created software is additionally conveyed and introduced in the acquirer end.

The important activities of a software process include software requirement gathering, Software design, Software construction, Software testing, Software maintenance, Software configuration management, Software engineering management, Software engineering process, Software engineering tools and methods, Software Quality Assurance and Risk management.

\section{SOFTWARE ESTIMATION MODELS}

So as to estimate effort, cost of software growth, many types of researchers proposed different approaches and these approaches are categorized into in subsequent ways [7]. 


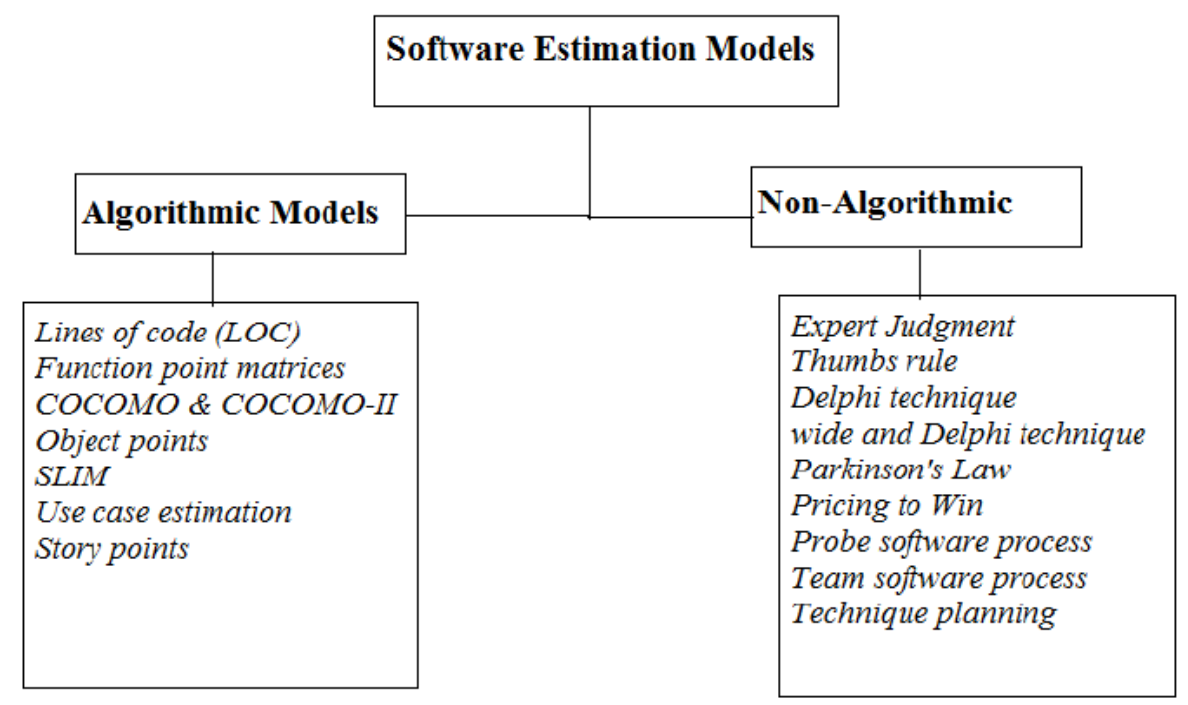

\section{SOFTWARE COST ESTIMATION TOOLS}

There are 21 commercial-off-the-shelf (COTS) cost estimation tools [9]. This set reflects a wide range of methodologies, levels of aptness or mellowness, features, and cost. Most of the tools are parametric models. Some tools address hardware as well as software, but most do not. A few tools offer a stochastic model. Developers of parametric models derive Cost Estimating Relationships (CERs) by regression analysis on historical data on project attributes (cost drivers) and cost. Cost estimation models use these relationships as scale factors in an exponential equation to calculate the effort and schedule required for the software development effort. The following 1 shows software cost estimation tools. 


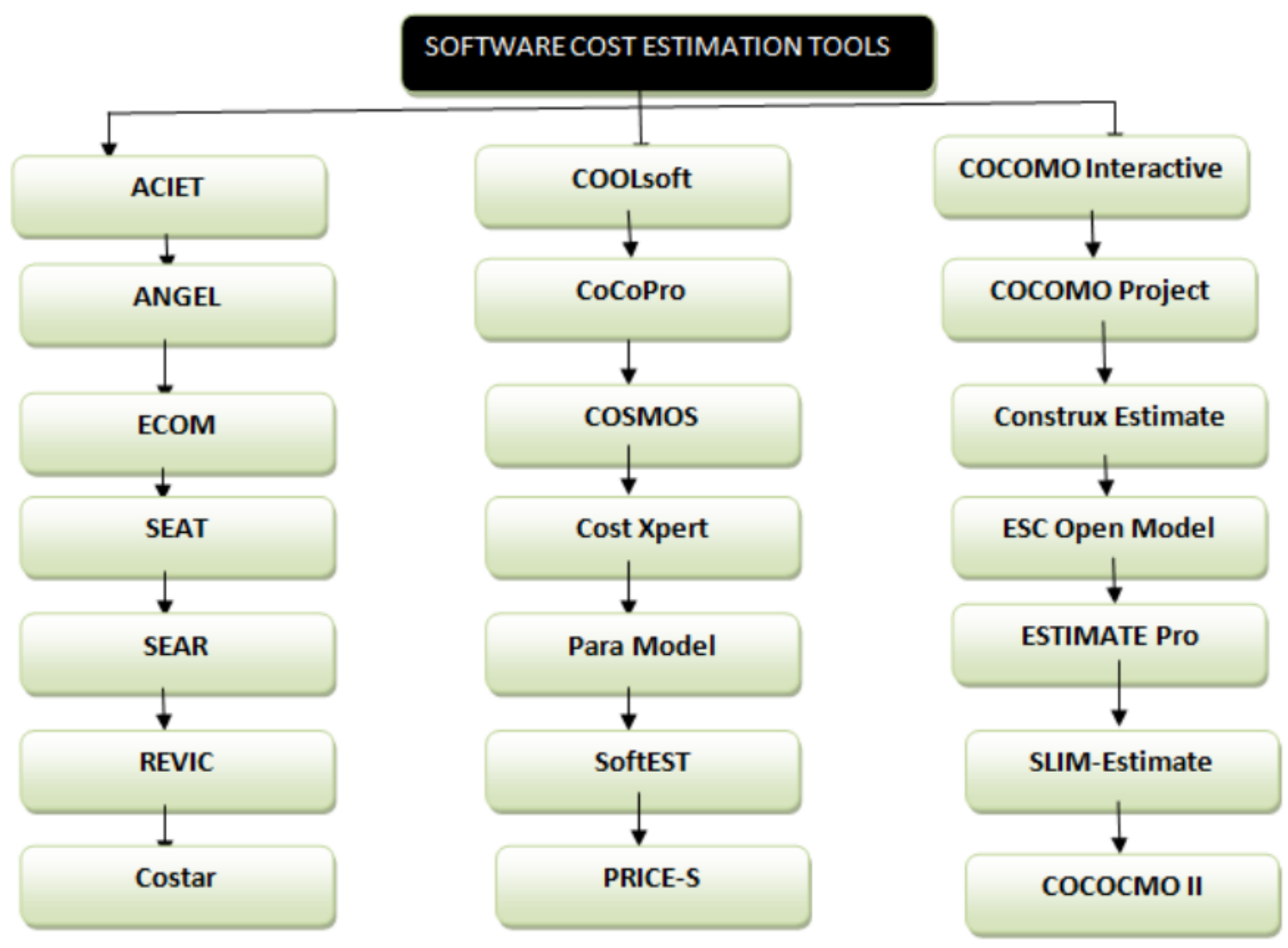

Figure 1 shows software cost estimation tools.

\subsection{Machine Learning Models}

For estimation of software Effort, duration and cost many algorithmic and non-algorithmic approaches are implemented but among this machine learning models are very accurate, these are machine learning models to predict software EDC.

\subsection{Fuzzy method}

The systems using this Fuzzy logic can simulate human behavior and reasoning. Fuzzy system tools efficiently solve the challenges in the situations like decision making is hard and huge number of constraints. These systems always takes the facts that ignored by other approaches. A fuzzy system consists of four steps:

Step 1: Fuzzification: It provides trapezoidal numbers for the linguistic terms.

Step 2: New linguistic term can be produced to determine complexity matrix.

Step 3: find out productivity rates and deduce linguistic terms.

Step 4: Defuzzification: estimate effort needed to complete the product and compare the previous techniques.

\section{CASE BASED REASONING}

Case based reasoning is a technique to collect and store the results of inspections on past products such as effort, language used, and cost so on. When we inspect the proposed product, if it identifies any new feature, then it is possible to recollect the store feature. 


\subsection{Assessment of CBR Systems}

CBR systems results are very interesting in terms of performance. CBR systems shows high performance compared to algorithmic approaches and close performance level to the expert. CBR systems used the stored database and have huge knowledge base to compare the new feature to stored one. Accurate estimates can be obtained by the CBR systems than experts. Experts don't have any capability to match their experience to the new observations.

\section{RULE-BASED SYSTEMS}

Rule based systems are very useful in estimating the effort of software in few cases. Rule based system stores some set of rules in its knowledge database, which have been used to compare the features of proposed project. When there is any identical rule is found, that rule will fire, which can be used to generate a new rule to form chaining effect. The chaining effect continues until there are no rules to fire and the result is displayed to user.

\section{REGRESSION TREES}

Regression trees are widely used to estimate the cost of the software in software engineering. A regression tree is constructed by investigating the attributes, and considers the most informative attributes. Use an algorithm on these informative attributes to construct the tree and split the attribute values where it is appropriate. Tree only ever splits into two leaf nodes. Checking can be done for any logical errors, by using this probable format.

\subsection{Artificial Neural Networks (ANNs)}

ANNs [6] are constructed based on the architecture of biological neural networks and these are also known as parallel systems. Artificial neuron is a collection networks that are interconnected. The neuron uses stepped function to determine the weighted sum of the inputs. The derived output might be a positive or negative given as input to other neurons in the parallel network. Feed-forward multilayer perception is one of the widely used models in ANN. Neurons are initialized with random weights. The network learns the associations between the inputs by altering the weights when the training set is obtainable from both the inputs and outputs. ANNs estimates development effort and it compares the accuracy to the algorithmic approaches rather than the suitability of this approach to estimation process. So many numbers of trials were required to found the number of concealed nodes. Other factors values are considered from experience and practical exercises.

\subsection{Genetic Programming}

Software estimation issue can be modeled as a symbolic regression equation. In regression model a set of values associated with the set of input factors, which generates an equation by considering these input factors as inputs to equation and it accurately estimates only one output factor. For example, a nearest neighbor system considers some of the factors to adjust the weights of variables and it discovers closeness of neighbors to get accurate result. The control parameters list used in genetic programming is being 20 and in neural networks its count is 10, but it is difficult to count control factors. However, sensitivity and accuracy does not depend on the count of the control factors. Granularity search is used to find probable values for control factors which will depend on their range also. To determine the ease of configuration for a genetic procedure, we can test the values of parameters. 


\subsection{Support Vector Machine (SVM)}

Support vector machines are used to perform classification and regression analysis by constructing hyper plane or various hyper planes in a high dimensional data space. The major goal of SVM is it discriminates two class labels, and it also reduces the error rate for the largest interval. The advantage of SVM is its assessment, by choosing acceptable parameters ' $C$ ' to construct optimal hyper plane. SVM constructs numerous hyper planes and chooses one of the hyper planes is optimal based on that it avoids local solution problem occurred in neural networks. SVM identifies the vectors which are intimately closed to known classes. This step is known as feature selection or feature extraction. These features accurately distinguish the classes or predict future samples. Learning process is very simple in SVM. There is no local optimal challenge like in neural networks. It scales well on a high dimensional space and there is proportional change of classifier complexity and error rate. Explicitly error rate will be controlled. Every data point can be viewed in dimensional space like a $\mathrm{p}$-dimensional point or vector with ( $\mathrm{p}-1)$ dimensional hyper plane.

\subsection{K-Nearest Neighbors (KNN)}

$\mathrm{K}$-nearest neighbor algorithm is classification method based on the training data set in the feature space. It is also known as instance based learning, or lazy learning. It uses function and it imprecisely estimates locally and all the calculations are delayed until to get the classifier. KNN algorithm is very simple among all other classification algorithms. In KNN an object is classified based on the majority votes of its neighbors, then the object being labeled the most frequent class name among its nearest neighbors where $\mathrm{k}$ is a positive integer. If $\mathrm{k}=1$ then that object is labeled by the neighbor class name because it has only one neighbor. Regression technique also uses same approach, but it labels the average value of the $\mathrm{K}$ nearest neighbors to the object. It is advantageous to assign the weights to each neighbor. A weight $1 / \mathrm{d}$ is assigned to each neighbor, where $\mathrm{d}$ is the distance from neighbor. This technique is known as linear interpolation. Neighbors should be considered from the set of objects, where the class labels must be known exactly.

\subsection{Bayesian Belief Networks (BBN)}

Bayesian Belief Networks based on graphical model and it shows the relationships between attributes. BBNs allow reasoning under uncertainty and take the benefits from both of the graphical representation and mathematical expressions in Bayesian probability. In BBNs, it is likely to eloquent beliefs of experts regarding variable dependencies and it proliferate the effect of facts on the uncertain outcomes, known as 'future system reliability'. BBNs also permit the insertion of technical rigidity when the probabilities of independent nodes are 'expert opinions'. BBN determines the inferences of the beliefs that are given as input factors. Some of the factors can be verified by using the previous project experimental results and experts experience. There are many benefits of using BBNs, one of the essential benefits is the facility to show and alter difficult models that are not developed by any other classical models. BBNs have meticulous mathematical derivations and the software tools are used to understand to do computations of their use. BBNs provide a better way to predict software flaws. BBN shows the join probability distribution among the factors. 


\subsection{Instance-Based Learning (IBL)}

The major challenge in software project management is the cost estimation. Most of the techniques used to estimate the cost and effort are based on the algorithmic models. Another technique to estimate the effort which is not based on the algorithmic model is known as instance-based learning. IBL approach gives high quality performance where the algorithmic model prediction is not possible.

\subsection{Decision Tree}

To make any decision it is very difficult with uncertain details because a) the significance outcome from choosing various specified decision choices cannot be estimated with certainty 2) So many different parameters should be considered to make a decision 3) It may be advantageous to think about the alternatives to reduce the uncertainty in the decision by adding some more values. 4) A decision maker's attitude headed to taking risk can affect the interest of various choices. A decision tree is a classification technique; it partitions the instance space iteratively.

\subsection{Statistical Approach for software EDC Estimation}

For estimation [11] of software EDC statistical SPSS Tool used by using this tool can find Mean, Mode, Standard Deviation and also can use T-Test, ANNOVA, Regression and Correlation testing.

\subsection{Data Source}

Kaggle.com [2] and PROMISE is an open source software Engineering repository where public datasets are available, using this can predict software models. For this experiment downloaded public data set from PROMISE and this data set contains following information like project id, project, Team experience, Manager Experience and Effort.

In SPSS tool [7] there are two types of views data view and variable view where in data view it shows data like in below figure 3 , which contain data mostly numeric.

\begin{tabular}{|c|c|c|c|c|c|c|c|c|c|c|c|}
\hline Prapar & Tomentip & Manspuexpe & Yowent & Law & Enen & Trencastiem: & Eention: & Fombetionadpait & Aquativent & Purtadpat & Langiage \\
\hline i & $\therefore$ & 4 & 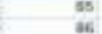 & 13 & 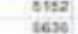 & 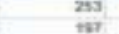 & 32 & ${ }_{300}^{360}$ & at & 30x & \\
\hline & 4 & 4 & 46 & I & 006 & 40 & so & $=0$ & til. & 83 & \\
\hline 4 & & 0 & at & s & 1022 & 200 & "19 & נות & 30 & 303 & \\
\hline s. & o & 9 & ac & 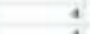 & rus & 30 & 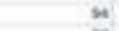 & 230 & 24 & 200 & \\
\hline 5 & 8 & s & as & 4 & Fast & $\pi$ & 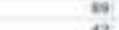 & 126 & a & 160 & 1 \\
\hline a & 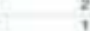 & 2 & $0_{0}^{85}$ & b & 政 & 19 & 42 & $x=1$ & 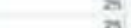 & is & \\
\hline 9 & 3 & , & 6 & a & tase & $m$ & w & 260 & >0 & $24 t$ & \\
\hline 10 & 3 & 4 & 93 & 4 & 262 & 78 & ; & 116 & 24 & 3 & \\
\hline 1 & 4 & , & M & 21 & 400 & 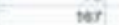 & $m$ & 200 & $z 4$ & 20 & \\
\hline 12 & 2 & 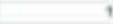 & is & 17 & gost & Me & 112 & 20 & 40 & $2 \pi$ & \\
\hline 13 & † & 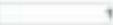 & as & 3 & 728 & na & $\eta$ & s] & 19 & $\omega$ & \\
\hline 14 & 3 & 4 & 48 & * & $41 / 2$ & $N e$ & t) & 273 & $x$ & 276 & \\
\hline is & & 4 & 85 & $\Rightarrow$ & & 223 & 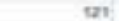 & 34 & $\Rightarrow$ & 100 & \\
\hline 16 & 3 & 2 & as & $\approx$ & 1617 & 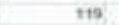 & 4I & $\$ 8$ & $\boldsymbol{x}$ & 16 & \\
\hline 17 & 4 & 3 & ${ }^{45}$ & * & nes & EI & 4 & 100 & 43 & 100 & \\
\hline 10 & & 4 & $\pi$ & 14 & sas? & en: & 310 & 36 & 20 & 156 & \\
\hline${ }_{n=1}^{10}$ & 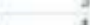 & 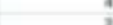 & 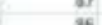 & 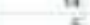 & 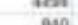 & $\therefore$ & 36 & $\infty$ & 21 & 3.0 & \\
\hline 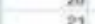 & & 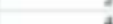 & 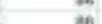 & $n$ & uave & 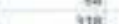 & 4 & 6 & 8 & & \\
\hline$z$ & 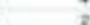 & 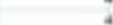 & 8 & 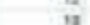 & 3 & 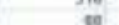 & 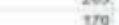 & 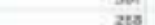 & 3 & 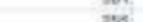 & \\
\hline 20 & 2 & 4 & 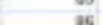 & $:$ & 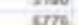 & 3e & (7) & 20 & $\hat{\imath}$ & w & 1 \\
\hline
\end{tabular}

Figure 3 SPSS data view 
An Implementation of Software Effort Duration and Cost Estimation with Statistical and Machine Learning Approaches

\begin{tabular}{|l|l|l||l|}
\hline \multicolumn{1}{|c|}{ Name } & \multicolumn{1}{c|}{ Type } & \multicolumn{1}{c|}{ Width } & Decimals \\
\hline id & Numeric & 12 & 0 \\
\hline Project & Numeric & 12 & 0 \\
\hline TeamExp & Numeric & 12 & 0 \\
\hline ManagerExp & Numeric & 12 & 0 \\
\hline YearEnd & Numeric & 12 & 0 \\
\hline Length & Numeric & 12 & 0 \\
\hline Effort & Numeric & 12 & 0 \\
\hline Transactions & Numeric & 12 & 0 \\
\hline Entities & Numeric & 12 & 0 \\
\hline PointsNonA & Numeric & 12 & 0 \\
\hline Adjustment & Numeric & 12 & 0 \\
\hline PointsAjust & Numeric & 12 & 0 \\
\hline Language & Numeric & 12 & 0 \\
\hline
\end{tabular}

Figure 4 SPSS variable view

In variable view it describes all variables with data type and width.

\begin{tabular}{|c|c|c|c|c|c|c|c|c|c|}
\hline & & \multirow[b]{3}{*}{ Frequency } & \multirow[b]{3}{*}{ Percent } & \multirow[b]{3}{*}{ Valid Percent } & \multirow{3}{*}{$\begin{array}{c}\text { Cumulative } \\
\text { Percent }\end{array}$} & \multicolumn{4}{|c|}{ Bootstrap for Percent ${ }^{\mathrm{a}}$} \\
\hline & & & & & & \multirow[b]{2}{*}{ Bias } & \multirow[b]{2}{*}{ Std. Error } & \multicolumn{2}{|c|}{ 95\% Confidence Interval } \\
\hline & & & & & & & & Lower & Upper \\
\hline \multirow[t]{7}{*}{ Valid } & -1 & 2 & 2.5 & 2.5 & 2.5 & .0 & 1.7 & .0 & 6.2 \\
\hline & 0 & 7 & 8.6 & 8.6 & 11.1 & -.1 & 3.3 & 2.5 & 16.0 \\
\hline & 1 & 20 & 24.7 & 24.7 & 35.8 & .0 & 5.0 & 14.8 & 34.6 \\
\hline & 2 & 18 & 22.2 & 22.2 & 58.0 & .0 & 4.6 & 13.6 & 30.9 \\
\hline & 3 & 13 & 16.0 & 16.0 & 74.1 & -.1 & 4.0 & 8.6 & 24.7 \\
\hline & 4 & 21 & 25.9 & 25.9 & 100.0 & .1 & 4.8 & 17.3 & 35.8 \\
\hline & Total & 81 & 100.0 & 100.0 & & .0 & 0 & 100.0 & 100.0 \\
\hline
\end{tabular}

Figure 5 Descriptive Statistics of Software

Fig 5 Shows Descriptive statics of software project and it shows cumulative percent, Bootstrap percents for given dataset. 


\begin{tabular}{|c|c|c|c|c|c|c|}
\hline \multicolumn{7}{|c|}{ Statistics } \\
\hline & & \multirow[b]{3}{*}{ Statistic } & \multicolumn{4}{|c|}{ Bootstrap ${ }^{b}$} \\
\hline & & & \multirow[b]{2}{*}{ Bias } & \multirow[b]{2}{*}{ Std. Error } & \multicolumn{2}{|c|}{$95 \%$ Confidence Interval } \\
\hline & & & & & Lower & Upper \\
\hline \multirow[t]{3}{*}{ Valid } & Project & 81 & 0 & 0 & 81 & 81 \\
\hline & TeamExp & 81 & 0 & 0 & 81 & 81 \\
\hline & Effort & 81 & 0 & 0 & 81 & 81 \\
\hline \multirow[t]{3}{*}{ Missing } & Project & 0 & 0 & 0 & 0 & $\overline{0}$ \\
\hline & TeamExp & 0 & 0 & 0 & 0 & 0 \\
\hline & Effort & 0 & 0 & 0 & 0 & 0 \\
\hline \multirow[t]{3}{*}{ Mean } & Project & 41,00 & .11 & 2.54 & 35.77 & 45.85 \\
\hline & TeamExp & 2.19 & .00 & .16 & 1.88 & 2.52 \\
\hline & Effort & 5046.31 & 10.80 & 509.16 & 4171.72 & 6208.23 \\
\hline \multirow[t]{3}{*}{ Median } & Project & 41.00 & .18 & 4.45 & 32.00 & 49.00 \\
\hline & TeamExp & 2.00 & .07 & .27 & 2.00 & 3.00 \\
\hline & Effort & 3647.00 & 27.94 & 324.47 & 2989.00 & 4277.00 \\
\hline \multirow[t]{3}{*}{ Mode } & Project & $1^{2}$ & & & & \\
\hline & TeamExp & 4 & & & & \\
\hline & Effort & $546^{3}$ & & & & \\
\hline \multirow[t]{3}{*}{ Std. Deviation } & Project & 23.527 & -.199 & 1.185 & 20.997 & 25.634 \\
\hline & TeamExp & 1.415 & -.011 & .079 & 1.245 & 1.560 \\
\hline & Effort & 4418.767 & .60 .068 & 642.377 & 3132.385 & 5628.299 \\
\hline
\end{tabular}

Figure 6 Statistics of given dataset

In Fig 6 shows statistical computation for software project in the way of number of projects, Team Exp and Effort required for project.

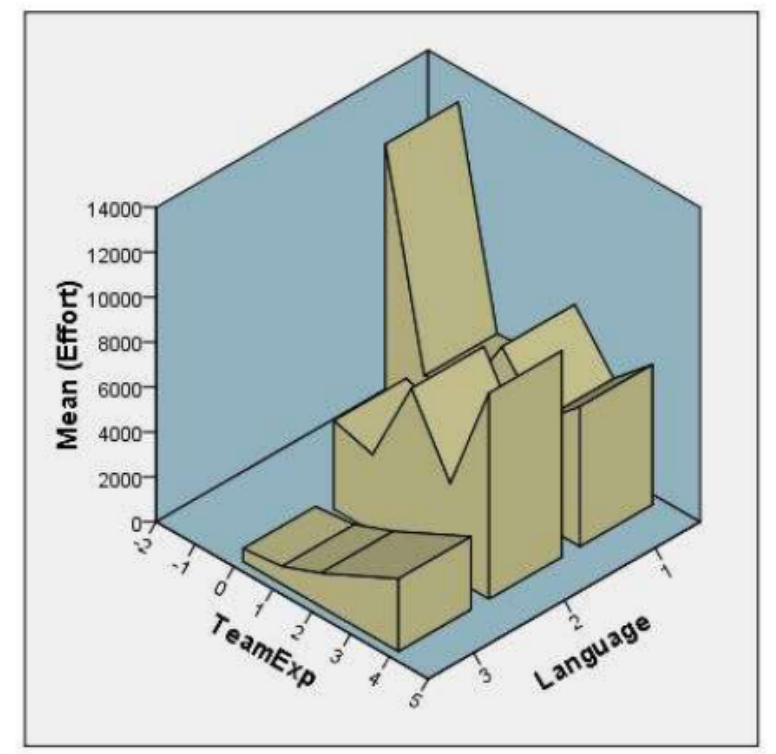

Graph 1 Software project statistical estimation

\section{PROJECT CODE METER FOR SOFTWARE EDC ESTIMATION}

It [1] is a software tool that allows project managers to measure and estimate the duration, cost, complexity, quality and maintainability of software projects and the productivity of the development team through their source code. Using a modern algorithm to determine the software, called Weighted Micro Function Points (WMFPs) [12], it follows strong ancestral 
An Implementation of Software Effort Duration and Cost Estimation with Statistical and Machine Learning Approaches

scientific methods such as COCOMO, COSYSMO, maintainability index, cyclomatic complexity and Halstead complexity and produces more accurate results than software tools .

\section{FUNCTIONALITIES OF CODE METER}

Below are functionalities which are supported by code meter: Outsourced work assessment, Remote employee monitoring, Software size comparison by development time (Effort), Budget Planning for software projects, Producing a Price Quote for source code, Predicting Development Schedule and Budget, Measuring past and ongoing Development Productivity, Evaluating the attractiveness of an Outsourcing offer (cost effectiveness), Assessing source code Quality and Obtaining source code Metrics.

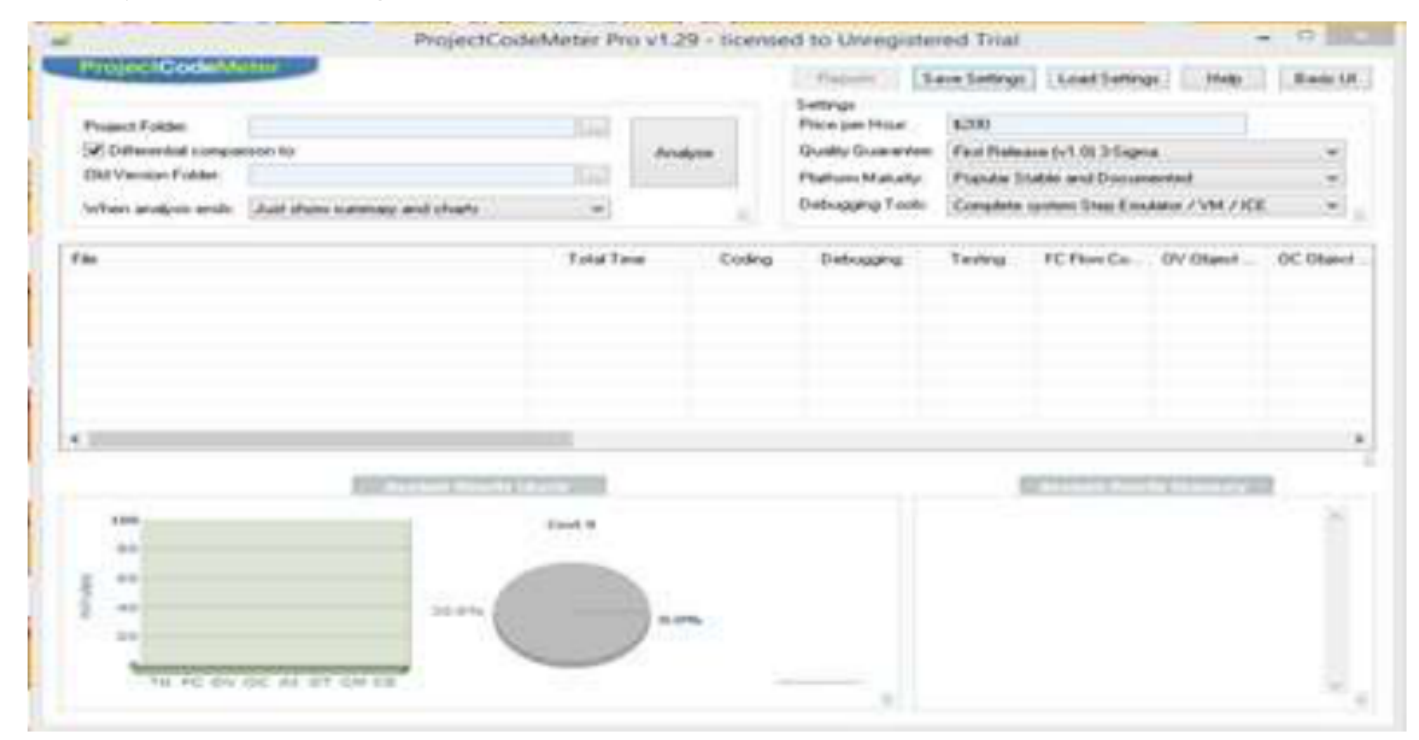

Figure 7 projectcodemeter home page

This is the home page where can give input as project folder and estimated hours it calculate and gives following output.

\begin{tabular}{||c|c||}
\hline \hline Settings & Normal \\
\hline \hline $\begin{array}{c}\text { Analysis } \\
\text { Type }\end{array}$ & $\$ 200$ \\
\hline \hline $\begin{array}{c}\text { Programmer } \\
\text { price per hour }\end{array}$ & First Release (v1.0) 3-Sigma \\
\hline \hline $\begin{array}{c}\text { Quality } \\
\text { Guarantee }\end{array}$ & Popular Stable and Documented \\
\hline \hline $\begin{array}{c}\text { Platform } \\
\text { Maturity }\end{array}$ & Complete system Step Emulator / VM / ICE \\
\hline \hline $\begin{array}{c}\text { Debugging } \\
\text { Tools }\end{array}$ & $\begin{array}{c}\text { F:ljavalprojects \Java_IEEE I2017\Detecting Mobile Malicious Webpages in } \\
\text { Real TimelcodelDetecting Mobile Malicious Webpages in Real Timeljs }\end{array}$ \\
\hline \hline Project Folder
\end{tabular}




\begin{tabular}{||c||c|c|}
\hline $\begin{array}{c}\text { Project Labor } \\
\text { Summary }\end{array}$ & \multicolumn{2}{|c|}{} \\
\hline \hline Total Time & 591 & Hours \\
\hline \hline Total Cost & $\$ 118277$ & \\
\hline
\end{tabular}

\begin{tabular}{|c|c|c|c|c|}
\hline \multirow[t]{2}{*}{ Statistical Labor Distribution } & & & & \\
\hline & Percent & Time in Hours & Time in Minutes & Cost in $\$$ \\
\hline Coding & 43 & 256 & 15406 & 51353 \\
\hline Debugging & 34 & 203 & 12191 & 40637 \\
\hline Testing & 22 & 131 & 7885 & 26283 \\
\hline FC Flow Complexity & 31 & 184 & 11095 & 36983 \\
\hline OV Object Vocabulary & 16 & 96 & 5763 & 19210 \\
\hline OC Object Conjuration & 30 & 179 & 10777 & 35923 \\
\hline AI Arithmetic Intricacy & 4 & 25 & 1518 & 5060 \\
\hline DT Data Transfer & 7 & 42 & 2536 & 8453 \\
\hline CM Comments & 0 & 0 & 20 & 67 \\
\hline CS Code Structure & 7 & 40 & 2418 & 8060 \\
\hline ID Inline Data & 4 & 22 & 1354 & 4513 \\
\hline Project Total & 100 & 591 & 35483 & 118277 \\
\hline
\end{tabular}

\begin{tabular}{||c||c||}
\hline \multicolumn{1}{|c|}{ Quality Measurements } & \multicolumn{1}{|c||}{} \\
\hline \hline Code Quality Notes Count & 7 \\
\hline \hline Code to Comment Ratio (CCR) & 1 \\
\hline \hline Essential Comment Factor (ECF) & 108 \\
\hline \hline Code Structure Modularity (CSM) & 37 \\
\hline \hline Logic Density (LD) & 27 \\
\hline \hline Source Divergence Entropy (SDE) & 1190 \\
\hline \hline Information Diversity Factor (IDF) & 31 \\
\hline Object Convolution Factor (OCF)
\end{tabular}


An Implementation of Software Effort Duration and Cost Estimation with Statistical and Machine Learning Approaches

\begin{tabular}{|c|c|c|c|c|c|c|}
\hline \multicolumn{6}{|c|}{ Quantitative Metrics } & \\
\hline \multicolumn{6}{|c|}{ Files } & 5 \\
\hline \multicolumn{6}{|c|}{ Logical Lines of Code LLOC } & 2573 \\
\hline \multicolumn{6}{|c|}{ Single Line Comments } & 10 \\
\hline \multicolumn{6}{|c|}{ Multi Line Comments } & 5 \\
\hline \multicolumn{6}{|c|}{ High Quality Comments } & 15 \\
\hline \multicolumn{6}{|c|}{$\begin{array}{c}\text { Strings } \\
\end{array}$} & 1859 \\
\hline \multicolumn{6}{|c|}{ Numeric Constants } & 1275 \\
\hline \multicolumn{7}{|c|}{35483} \\
\hline \multicolumn{7}{|c|}{$11095 \quad 10777$} \\
\hline & & 570 & & 1518 & 2530 & $20 \quad 2418$ \\
\hline Tossi & $\mathrm{FC}$ & ov & $o c$ & A & का & $\overline{\mathrm{CM}} \mathrm{cs}$ \\
\hline
\end{tabular}

Figure 8 analysis of software estimation

\section{CONCLUSION}

In this paper first we studied Machine learning models, other estimation models and tools for software estimation and analyzed Software EDC with statistical tool (SPSS) and Software cost estimation tool (Project code Meter), in early literature most of the researcher's implemented human judgment and other approaches for EDC estimation but these are not accurate in practical, in the second stage many researchers focused on statistical approaches, these approaches need strong statistical knowledge and it produce semi accurate result. To this Project code meter is estimation tool where it shows complete EDC estimation with accurately.

\section{REFERENCES}

[1] http://www.projectcodemeter.com/cost_estimation/kop1.html ProjectCodeMeter

[2] https://www.kaggle.com/raulvilla/software-cost-estimation-from-promise public dataset

[3] Aprna Tripathi, Bhuvnesh Kumar, Ashish Sharma, Dharmender Singh Kushwaha, "SRS Based Estimation of Software Maintenance Effort", 2012 Third International Conference on Computer and Communication Technology, pp.154-155, 2012.

[4] Barry W. Boehm, "Software Cost Estimation Meets Software Diversity", 2017 IEEE/ACM $39^{\text {th }}$ International Conference on Software Engineering Companion (ICSE-C), pp. 495-496. 2017.

[5] Chin-Yu Huang \& M.R. Lyu, "Optimal release time for software systems considering cost, testing-effort, and test efficiency", Volume 54, Issue 4, pp.583-591, 2005

[6] Farhad Soleimanian Gharehchopogh, "Neural networks application in software cost estimation: A case study", International Symposium on Innovations in Intelligent Systems and Applications, pp.69-73. 2011

[7] Jacky Wai Keung, Barbara A. Kitchenham, David Ross Jeffery, "Analogy-X: Providing Statistical Inference to Analogy-Based Software Cost Estimation, Volume 34, Issue 4, pp.471-484. 2008 
[8] M. Jorgensen \& K. Molokken-Ostvold, "Reasons for software effort estimation error: impact of respondent role, information collection approach, and data analysis method", Volume 30, Issue 12, pp.993-1007. 2004

[9] Shahab Nadir, Detlef Streitferdt, Christina Burggraf, Industrial Software Developments Effort Estimation Model”, 2016 International Conference on Computational Science and Computational Intelligence (CSCI), pp.1248-1252, 2016.

[10] T. Gruschke, "Empirical studies of software cost estimation: training of effort estimation uncertainty assessment skills", $11^{\text {th }}$ IEEE International Software Metrics Symposium (METRICS'05), pp.3-48. 2005

[11] Tan Chin Hooi, Yunus Yusoff, Zainuddin Hassan, "Comparative Study on Applicability of WEBMO in Web Application Cost Estimation within Klang Valley in Malaysia", 2008 IEEE $8^{\text {th }}$ International Conference on Computer and Information Technology Workshops, pp.116-121. 2008

[12] Yinhuan Zheng, Beizhan Wang, Yilong Zheng, Liang Shi, 2009, Estimation of software projects effort based on function point", $20094^{\text {th }}$ International Conference on Computer Science \& Education, pp.941-943. 2009.

\section{ABOUT AUTHORS}

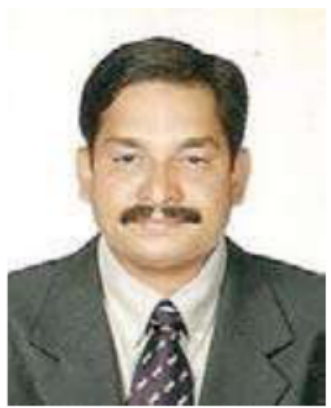

Dr. P.V.S. Srinivas is presently serving as a Professor in Computer Science and Engineering, at Srinidhi Institute of Science and Technology, Hyderabad, Telanagana. He has got his Masters followed by Ph.D. in computer Science and Engineering in the area of Computer Networks from JNTU Hyderabad in the year 2003 and 2009 respectively. His main research interests are Wireless Communication, Mobile Computing and Mobile Ad hoc Networks. His research focuses in on "Designing an Effective and Assured Communication in MANETs" and improving QoS in MANETs. He is also serving as a Chief Panel Consultant in the area of wireless communications for a Hyderabad based company by name a SCADA METER SOLUTIONS Pvt Ltd. He has published 32 research papers in different refereed International Journals and conferences in India as well as abroad. $\mathrm{He}$ is also serving as an Editor-in-Chief for an International Journal IJWNC and also a peer reviewer for 3 International Journals.

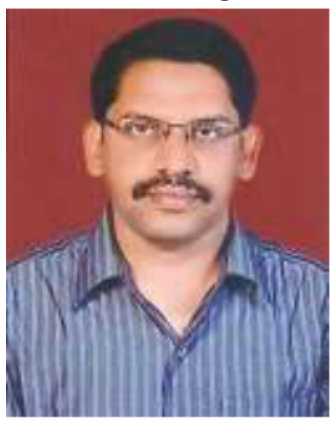

Mr. B.M.G. Prasad presently serving as Professor in Computer Science and Engineering at Holy Mary Institute of Technology and Science, Hyderabad, Telangana. He is having 18 years of teaching experience in various Engineering Colleges. He has done his B.Tech at Vijaya Nagar Engineering College, Bellary in 1997. He completed his M.Tech at J.N.T.U College of Engineering, Anatapur in 2003 and now he is persuing his Ph.D in Computer Science and engineering under the guidence of Dr. P.V.S. Srinivas,, in RAYALASEEMA UNIVERSITY, KURNOOL A.P State. He has published papers in the I-Manager's International Journal and Indian National Science Congress, Tiruvananthapuram in the Computer Networks area. And his areas of interest are Software Engineering, Wireless Networks, Image Processing etc. 